\title{
Diabetische Polyneuropathie
}

\section{Benfotiamin-Gabe - eine pathogenetisch orientierte Therapie}

Bei schlechter Diabeteseinstellung sind bereits leicht erhöhte Blutzuckerspiegel toxisch für die Nerven. Eine Schädigung der Nervenfasern tritt deshalb schon früh im Krankheitsverlauf auf, oft bereits im Stadium des Prädiabetes, erklärte Prof. Dan Ziegler, Düsseldorf.

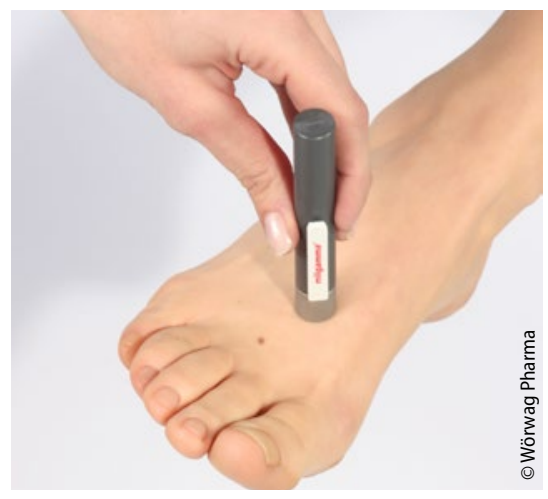

An Anfang steht die Einbuße des Vibrationsempfindens.
Patienten mit diabetischer Neuropathie, die häufig an Schmerzen oder Missempfindungen in den Füßen leiden, sollten deshalb frühzeitig adäquat behandelt werden. Prinzipiell gibt es dafür drei Möglichkeiten, betonte Prof. Kristian Rett, München. Neben einer individualisierten Diabetestherapie kann eine symptomatische Therapie die Beschwerden lindern.

Von besonderer Bedeutung sei jedoch die frühzeitige, pathogenetisch orientierte Behandlung mit der hoch bioverfügbaren, lipidlöslichen Vitamin$\mathrm{B}_{1}$-Vorstufe Benfotiamin (milgamma protekt). Damit lassen sich bei der Glukose-Verarbeitung die Produktion schädlicher Abfallprodukte - z. B. Advanced Glycation End-products (AGE) und damit Nerven- und Gefäßschäden vermeiden.

Wie Rett erläuterte, leitet Benfotiamin durch die Aktivierung des thia- minabhängigen Enzyms Transketolase die überschüssige Glukose in den unschädlichen Phentosephosphat-Shunt um und verhindert damit das Überlaufen in die vier pathogenen Stoffwechselwege der Glykolyse (Polyol-, Hexosamin-, Proteinkinase-C- und AGE-Stoffwechselweg).

\section{Schmerzen werden eingedämmt}

Dass Benfotiamin schmerzhafte und sensorische Symptome der diabetischen Neuropathie verbessern kann, belegen verschiedene kontrollierte Studien (u. a. Haupt E et al. Int J Clin Pharmacol Ther. 2005;43:71-7; Stracke H et al. Exp Clin Endocrinol Diabetes. 2008;116:600-5). Die stärksten Effekte zeigten sich dabei beim Symptom Schmerz.

\section{Dagmar Jäger-Becker}

- Pressekonferenz „Diabetes! Hören Sie auf Ihre Füße?“; Berlin, Mai 2016 (Veranstalter: Wörwag)

\section{Antikoagulation in Sekunden aufgehoben}

\section{Antidot für Dabigatran verfügbar}

— Für das nicht-Vitamin-K-abhängige neue orale Antikoagulans (NOAK) Dabigatran ist sowohl durch die Phase-IIIZulassungsstudie RE-LY ${ }^{\circledast}$ (Randomized Evaluation of Long term anticoagulant therapY) als auch im Rahmen der Anwendung im klinischen Alltag ein günstiges Nutzen-Risiko-Profil belegt. Das berichtete Prof. Harald Darius, Berlin.

Jetzt wurde mit Idarucizumab (Praxbind ${ }^{\star}$ ) auch noch ein spezifisches Antidot zur Aufhebung der gerinnungshemmenden Wirkung von Dabigatran für seltene kritische Notfallsituationen entwickelt. Idarucizumab ist ein voll humanisiertes Antikörperfragment, das intravenös appliziert wird.

Das Antidot bindet selektiv gebundenes und freies Dabigatran im Blutstrom. „Es besitzt keine prokoagulatorische oder antikoagulatorische Wirksamkeit und führt innerhalb von Sekunden zur vollständigen und anhaltenden Aufhebung der Wirkung von Dabigatran“, erläuterte Darius [Pollack CV et al. Idarucizumab for Dabigatran Reversal. N Engl J Med. 2015;373:511-20]. Dank des Antidots seien Notfalleingriffe einfacher durchführbar, wodurch insgesamt ein neues Sicherheitsniveau in der gerinnungshemmenden Therapie erreicht werde.

Dieser Ansicht stimmte Prof. Martin Grond, Siegen, zu: „Wir sehen immer wieder antikoagulierte Patienten, die einen ischämischen Schlaganfall erleiden. Früher hätten wir uns niemals getraut, diese Patienten zu lysieren.

\section{Dr. Silke Wedekind}

- Interdisziplinäres Pressegespräch „Erste Erfahrungen mit dem Pradaxa ${ }^{\oplus}$-spezifischen Antidot: Praxbind ${ }^{\oplus}$ in der Praxis"; Frankfurt/M., September 2016 (Veranstalter: Boehringer Ingelheim)

\section{Kurz notiert}

Mehr auf HIV testen! $\rightarrow$ Um die HIV-Epidemie einzudämmen, hat das Joint United Nations Programme on HIV/AIDS 2014 einen Plan mit dem Titel "90-90-90“ vorgestellt: Bis 2020 sollen weltweit $90 \%$ aller Menschen mit HIV von ihrer Infektion wissen. Davon sollen $90 \%$ eine HIV-Therapie erhalten, von denen wiederum bei $90 \%$ die Viruslast dauerhaft unter der Nachweisgrenze liegt.

Deutschland steht bei der Erreichung des Ziels gut da, aber auch hierzulande wissen nur $84 \%$ der HIV-positiven Menschen, dass sie HIV-infiziert sind. Auf dem Symposium „On the road to 90-90-90" stellte ViiV Healthcare sein Engagement vor, um hierzulande zu dem Programm beizutragen. Ein Schwerpunkt lag dabei auf der Verbesserung der Akzeptanz der HIV-Testung. „Denn nur wer von der eigenen Infektion weiß, kann die Vorteile einer antiretroviralen Therapie nutzen - für sich und auch für andere", resümierte Sonja Luz, ViiV Healthcare.

Red.

- Nach Informationen von ViiV 